\title{
Understanding beam alignment in a coherent lidar system
}

\author{
Narasimha S. Prasad* \\ NASA Langley Research Center, 5 N. Dryden St., MS 468, Hampton VA, 23681 \\ Chandrasekhar Roychoudhari \\ University of Connecticut, Storrs, CT 06269
}

\begin{abstract}
Optical beam alignment in a coherent lidar (or ladar) receiver system plays a critical role in optimizing its performance. Optical alignment in a coherent lidar system dictates the wavefront curvature (phase front) and Poynting vector) matching of the local oscillator beam with the incoming receiver beam on a detector. However, this alignment is often not easy to achieve and is rarely perfect. Furthermore, optical fibers are being increasingly used in coherent lidar system receivers for transporting radiation to achieve architectural elegance. Single mode fibers also require stringent mode matching for efficient light coupling. The detector response characteristics vary with the misalignment of the two pointing vectors. Misalignment can lead to increase in DC current. Also, a lens in front of the detector may exasperate phase front and Poynting vector mismatch. Non-Interaction of Waves, or the NIW property indicates the light beams do not interfere by themselves in the absence of detecting dipoles. In this paper, we will analyze the extent of misalignment on the detector specifications using pointing vectors of mixing beams in light of the NIW property.
\end{abstract}

Key words: Coherent lidar system, optical beam alignment, Poynting Vector

\section{THE NON-INTERACTING OF WAVES (NIW) PROPERTY}

In the absence of any 'obstructing' detectors, all the Huygens-Fresnel secondary wavelets will continue to propagate unhindered and without interacting (Interfering) with each other. Light beams do not interfere by themselves. The superposition effects due to light beams become manifest through the response characteristics of the detecting dipoles. Accordingly, all the properties that we generally attribute to only light, are in reality manifestations of collective properties of dipole-light interactions. "Interference" and "coherence" can be better understood in terms of this mutual interaction, followed by energy absorption by the dipoles from EM wave fields, manifesting in some measurable transformation of the detecting dipoles. The NIW property states that in the linear domain, all waves pass through each other unperturbed [1]. Different harmonic undulations of the same tension field cannot exert any force of interaction on each other. Otherwise the above observations would not be possible. Well formed light beams cross each other without modifying each other's spatial and/or temporal energy distribution. EM waves do not interact with each other. We have even named "photons" as Bosons. Figure 1 illustrates four scenarios that acknowledge the NIW-Property of NonInteraction of Waves in nature. In general, we have been ignoring the absence of any physical interaction process (force) between waves! In reality, the subtlety of non-interference of wave forms is a generic property of nature!

In the light of NIW property, there is a need to understand the nature of misalignment in coherent lidar systems [2]. We generally accept the experimentally observed criteria for heterodyne detections that the two waves that are mixed must (i) be collinear, (ii) have matched wave fronts and (iii) cannot be orthogonally polarized. We have not found in the literature adequate physical explanations for these requirements. The purpose of this paper is to find deeper physical understanding of the coherent heterodyne detection processes that could lead to better coherent laser radar system designs. The dependence of signal-to-noise ratio is critical on the optimal alignment of heterodyne signals at the detector in a coherent lidar system. Optical alignment in a coherent lidar system dictates the wave front curvature (phase front and Poynting vector) matching of the local oscillator beam with the incoming receiver beam on a detector. However, this alignment is often not easy to achieve and is rarely perfect. Furthermore, optical fibers are being increasingly used

\footnotetext{
*narasimha.s.prasad@nasa.gov; Phone 757-864-9403; Fax 757-864-8828.
} 
in coherent lidar architectures, which have their own perfect mode matching requirements that are non-trivial. In this paper, we will explore misalignment issues using Pointing vector formulation.

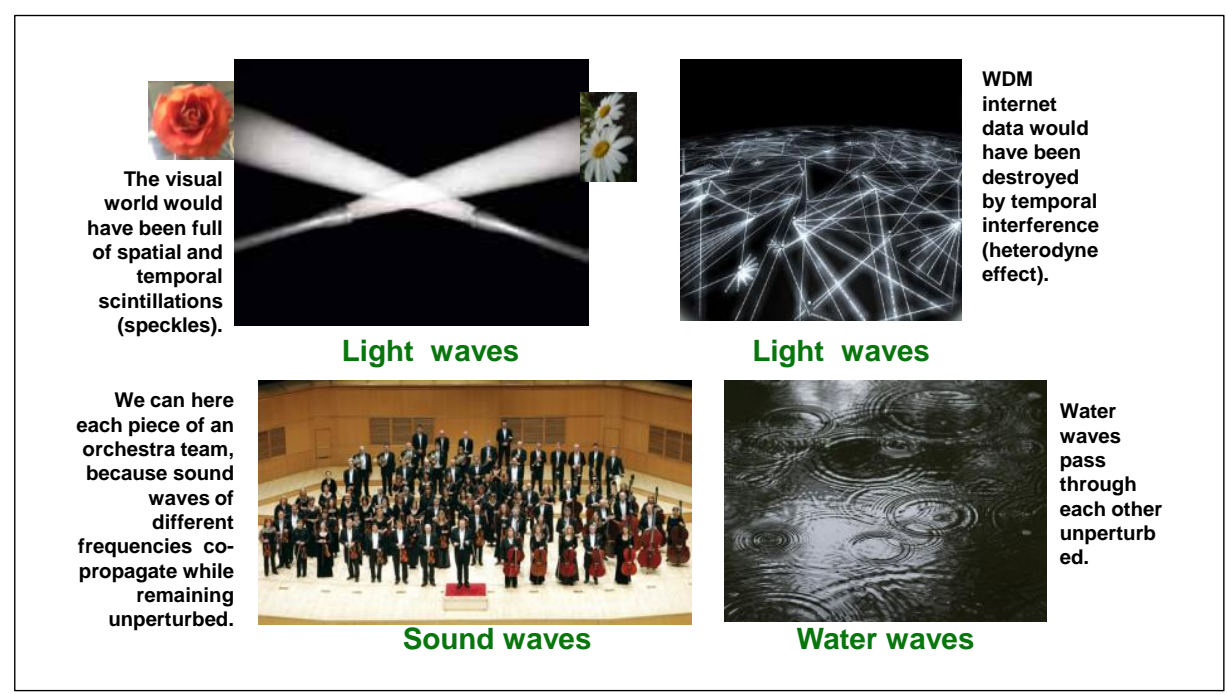

Figure 1. Four scenarios illustrating the NIW property. Top left: The fidelity of the visual world remains unperturbed even though all optical beams are always crossed by innumerable other beams. Top Right: Spectral and Doppler shift information carried by any specific star light remains unperturbed by billions of other crossing star lights. Bottom Left: NIW property in the acoustics domain and co-propagation of acoustic waves without interference. We can discern all the different frequencies from different instruments. Bottom Right: The NIW property exhibited by water waves. Water waves pass through each other without perturbing each other's original propagation behavior!

\section{Understanding detectors' roles behind measured superposition effects :}

Scientific data are measurement of re-producible quantitative transformations that are experienced by our interactants (or detector-detectee, or sensor-sensee). Any transformation in measurable physical parameters requires energy exchange between the interactants. The energy exchange must be guided by a force of interaction between the interactants and it must be strong enough to facilitate the exchange of energy, which are usually constrained by the characteristic limitations of each interactant. All force rules being distance dependent, energy exchange between the interactants requires that they must experience each other as locally present or physically superposed entities (experience each other within their sphere of influence). Interactants must be physically superposed within the range of the operating interacting force that will allow some energy exchange followed by some transformations that is measurable data for us. Superposition effect is an active local process, not a passive mathematical principle! While the observable material and light based universe may generically appear as NON-LOCAL, a careful analysis of "SUPERPOSITION EFFECTS as MEASURED DATA" tells us that all measured phenomena are necessarily LOCAL since all forces of interactions have a finite physical range! All mathematical formulations must recognize this Reality Ontology [3].

There are several related fundamental questions of interest. They are: (i) Why does the temporal fringes due to superposition of different frequency beams die out rapidly with non collinearity but the spatial fringes due to superposition of same frequency beams never die out with non collinearity? (ii) Why longitudinal laser modes (frequencies) appear as "incoherent" in a Michelson Fourier transform spectrometer (FTS) but as "coherent" on a high speed detector? (iii) What is the difference between "temporal coherence" and "spectral coherence"? (iv) Why does orthogonally polarized light do not "interfere"? Are they "incoherent"? (v) Can coherence be really defined as fieldfield correlation? These questions have to be analyzed in relation to the NIW property to obtain new insights into the behavior of light. Light beams do not "interfere" or generate energy re-distribution (fringes) by themselves! It is the quantum properties of the detecting dipoles that dictate which joint stimulations induced by the superposed fields can be summed. Appreciating the NIW-property and the role of detector in carrying out the measurable superposition effects will enable us to unlock the deeper nature of photons. 


\section{MISALIGNMENT IN HETERODYNING PROCESSES}

This paper addresses two major optical alignment issues critical for efficient coherent lidar systems: (i) Non collinearity of the local oscillator (LO) and the signal wave-fronts and (ii) mismatch between their phase-fronts. Figure 2 illustrates these two situations of mismatch.

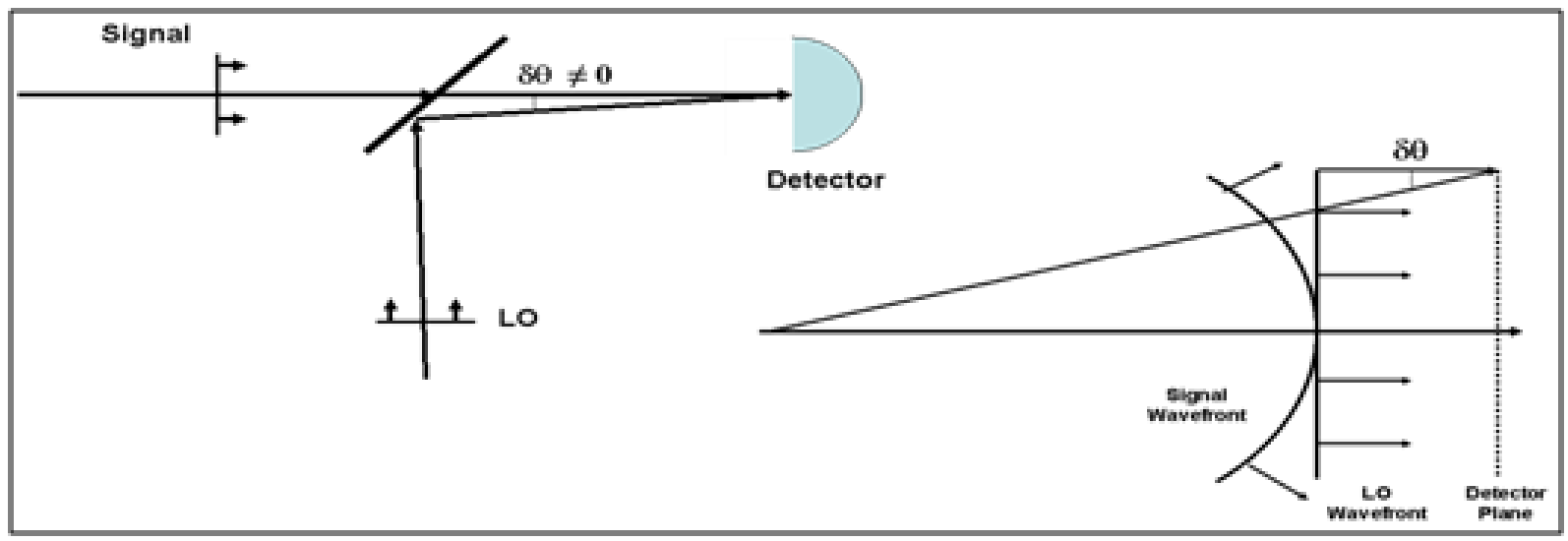

Figure 2. Non collinearity of the LO and signal wave fronts and mismatch of their phase fronts are inseparable. Left: Misalignment due to non-collinearity. Right: Phase Mismatch of heterodyning signals at the detector.

\section{SUPERPOSITION OF TWO LIGHT BEAMS}

To understand the effects of misalignment, let us analyze crossing of two light beams of same frequency as illustrated in Figure 2 [4]. In Figure 3, origin of spatially and temporally shifting fringes due to same frequency waves is examined. A pair of phase-steady collimated Gaussian laser beams is crossing through each other, as in Figure 3(a). The relative phase delay between the two beams along the horizontal X-axis can be computed using the diagram in Figure 3(b). Mathematically, the complex and real parts of resultant of two superposing EM waves are shown in Equation 1.

$$
\begin{aligned}
E_{c x .}(t, \tau) & =a e^{i 2 \pi \nu(t-\tau / 2)}+a e^{i 2 \pi \nu(t+\tau / 2)} \\
E_{r l .}(t, \tau) & =a \cos 2 \pi v(t-\tau / 2)+a \cos 2 \pi \nu(t+\tau / 2) \\
& =2 a \cos 2 \pi v t \cos \pi v \tau
\end{aligned}
$$

This elementary conceptual experiment helps us appreciate the difference between mathematical superposition principle and measurable superposition effects that our optical detectors report. 


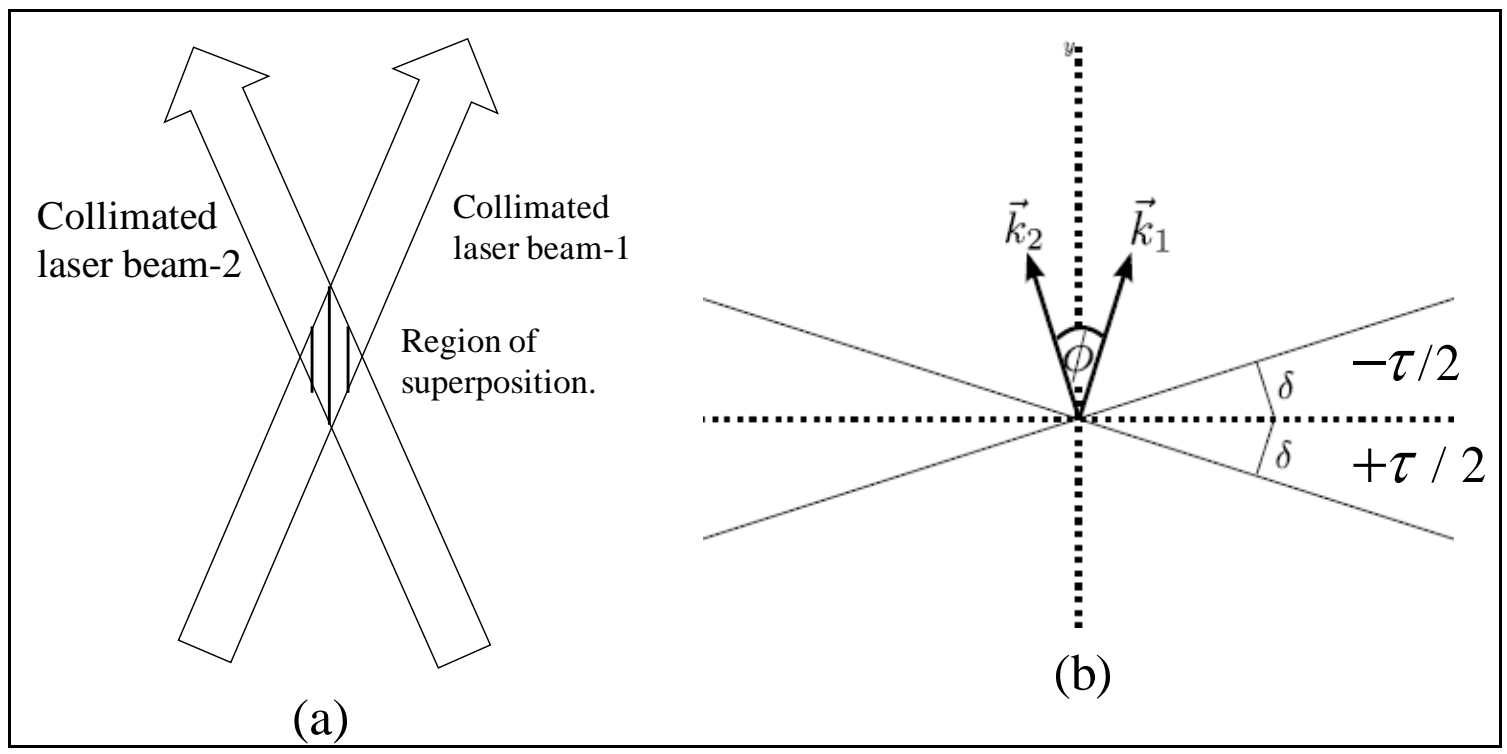

Figure 3. Superposition of two collimated EM waves. (a) Region of superposition will have fringes.

(b) Relative phase delays experienced by two superposing EM waves.

Figure 4 illustrates the nature of fringes of two beams crossing each other. Time averaged fringes (as in a), which we normally register in the optical domain. But time varying real representation (as in b) tells us that the intensity in the bright fringe region is oscillating at the optical frequency. The square modulus of the complex representation directly gives the time averaged fringes we usually record by optical detector arrays. The third plot (c) shows the case when the two beams carry different frequencies. Then fringes also move laterally in the space domain (as in the case of a heterodyne coherent lidar). From the NIW property, the detector carries out the joint dipolar stimulations before absorbing the energy from both the fields. The "summing" role of a detector becomes obvious from its intrinsic "time averaging" property, which cannot be executed by EM waves. As such, close attention has to be paid towards the role of detector in maximizing the signal to noise ratio

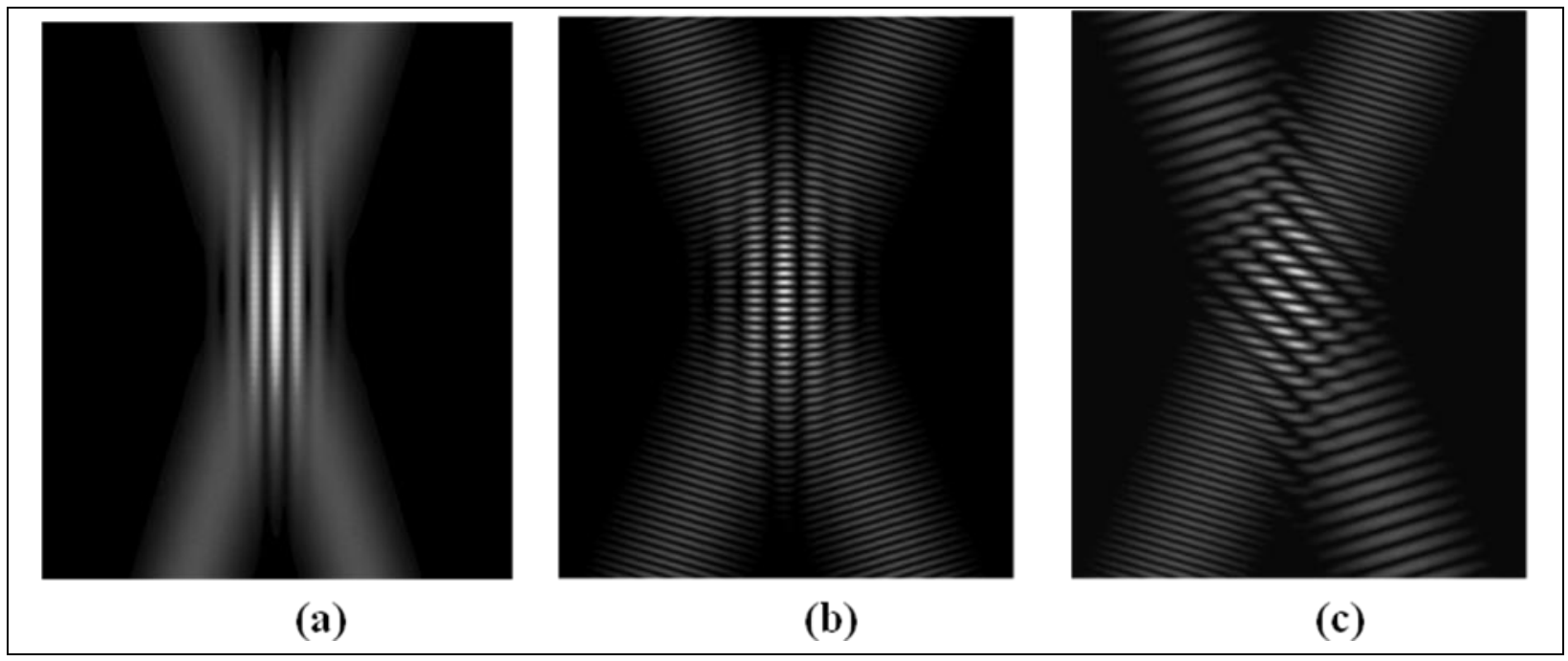

Figure 4. Fringes due to crossing of two light beams. (a) Time averaged fringes. (b) Instantaneous time varying fringes. Time varying real representation in which fringes appear stationary. (c) Time varying fringes due to crossing of light beams of two different frequencies. In this case fringe motion is observed. 
Figure 5 illustrates mathematically the result of superposition of two beams for cases (a) and (b) shown in Figure 2. $\mathrm{E}_{\mathrm{rl}}$ represents the real electric field amplitudes, $\mathrm{E}_{\mathrm{cx}}$ represents the complex amplitude, $v$ represents the optical frequency, $t$ is the time, $\tau$ is the time delay between the two beams, $\chi$ is the susceptibility, $I_{r l}$ is the real intensity of resulting beams and $\mathrm{I}_{\mathrm{cx}}$ is the complex intensity of resulting beams. In this analysis, $\chi$ is the susceptibility factor that describes the nonlinearity of the medium. Upon analyzing the resultant of superposition for the two cases of instantaneous time varying fringes and time averaged fringes, we see a factor of 2 difference. The "summing" role of a detector becomes obvious from its intrinsic "time averaging" property, which cannot be executed by EM waves.

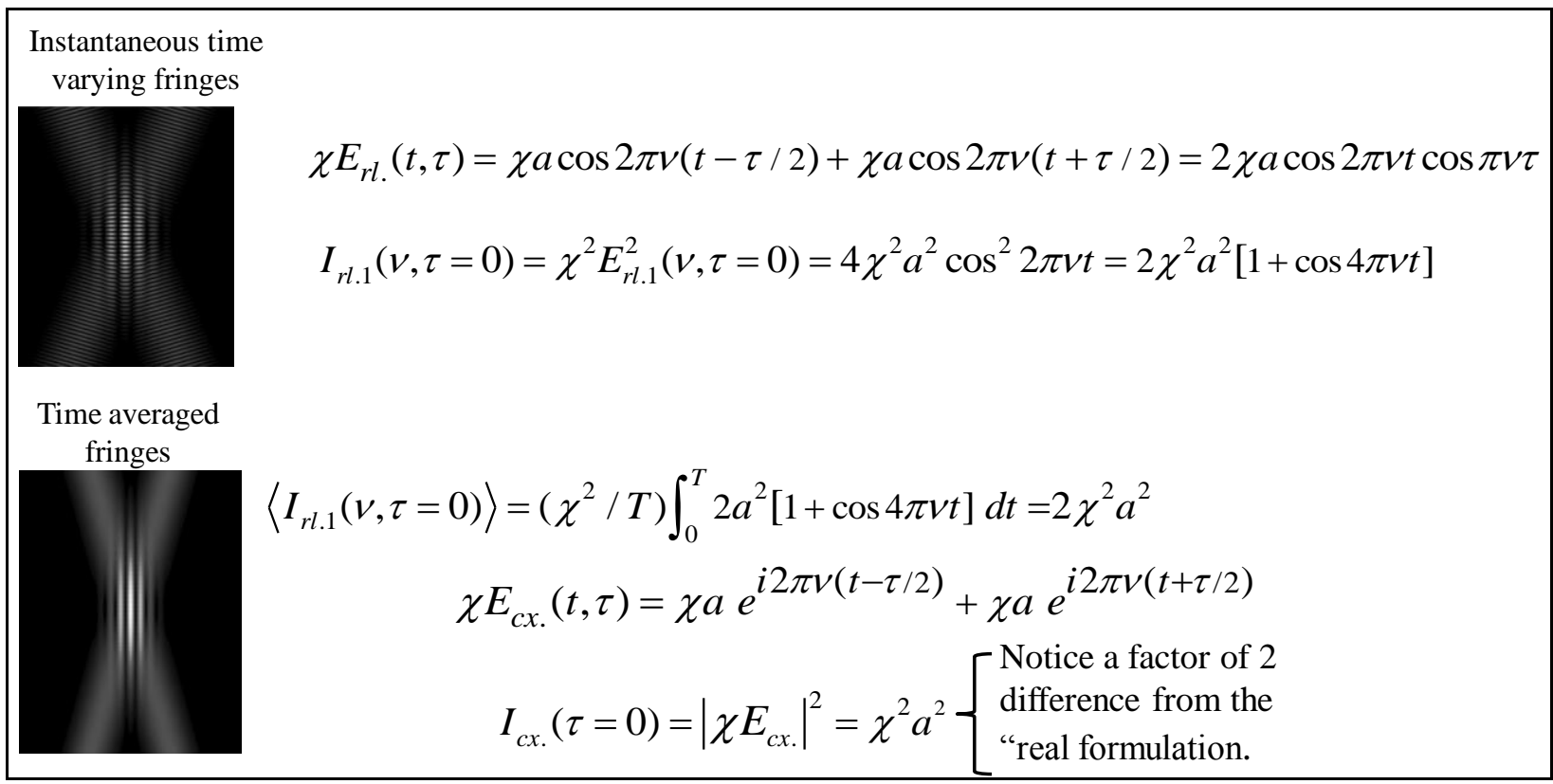

Figure 5. Mathematical analysis of instantaneous time varying fringes and time averaged fringes. A factor of 2 difference for real and complex formulations can be noted [5].

\section{Comparison of Michelson FTS and heterodyne detection validates NIW property:}

In heterodyne detection, modern fast detectors are able to pickup time varying heterodyne signals. Michelson Fourier transform spectroscopy is done using very slow detectors which averages out the time varying signal. Thus, we see again the detectors determine the superposition effect based on their temporal responses. Waves are not interfering to produce intensity variations by themselves (NIW property)

\section{ANGULAR MISALIGNMENT OF LIGHT BEAMS}

Let us now consider the effect of left-over misalignment or non-collinearity of the Poynting vectors between the signal and the reference beams. Figure 6 illustrates the role of physical size of the detector in relation to the angular misalignment and its effect on the system signal to noise ratio. The arrow indicates the direction of the spatial velocity of fringes due to reduction in angular misalignment. In Figure 6, the left most picture illustrates the averaging over space in relation to the detector size. The deleterious effect on the signal to noise ratio can be inferred from this. As the angular misalignment reduces, lateral averaging of signal drops away and the temporal oscillation dominates over spatial averaging. We want zero spatial fringe movement to record pure temporal heterodyne signal oscillations. This maximizes the signal to noise ratio seen by the detector.

Collinearity requirement to measure heterodyne frequencies was carried out and is illustrated in Figure 7 [6]. In this experiment, temporal modes from two He-Ne laser beams were used as two heterodyning beams. An electronic spectrum analyzer recorded the signal strengths as the angular misalignment was varied. The angular misalignment was 
obtained using a glass block mounted on a rotational stage. Heterodyne signal strength rapidly decreases as the angle between the superposed beams deviates from zero.

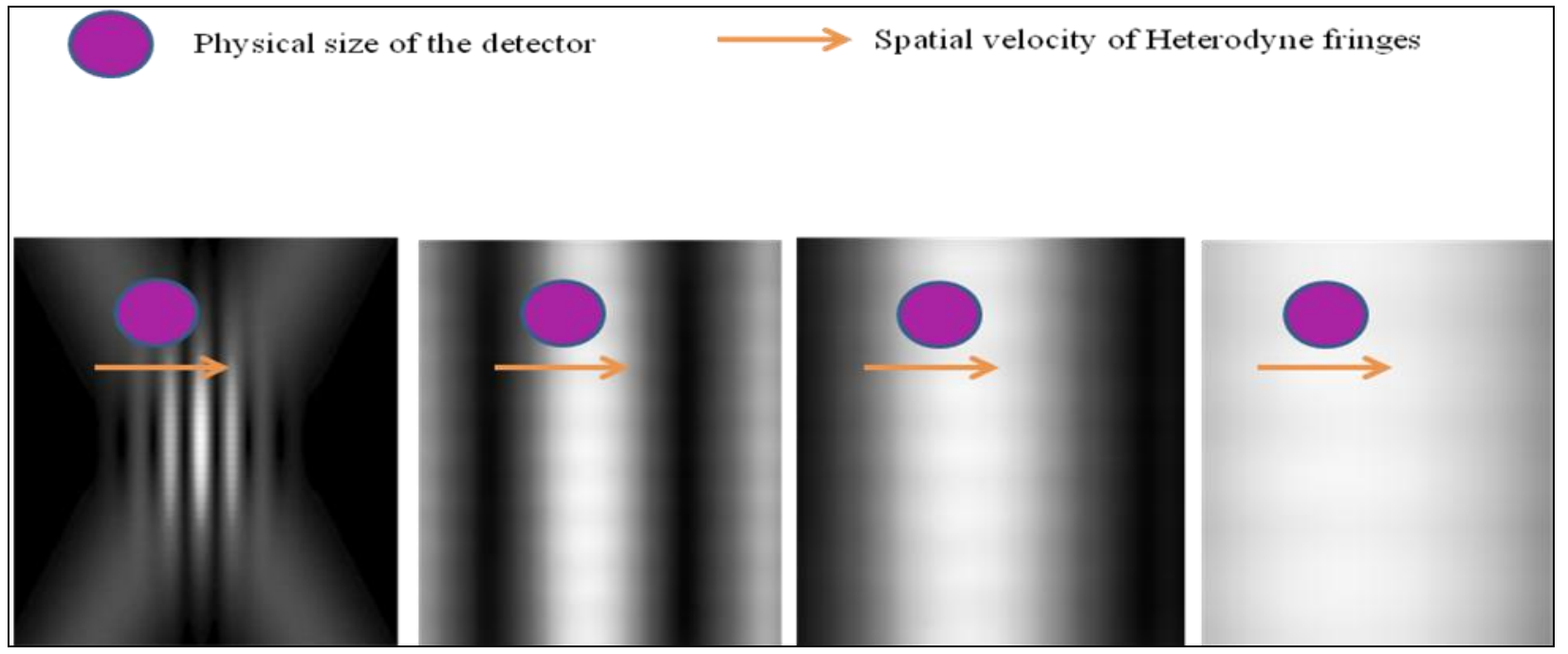

Figure 6. The effect of fringes arising due to angular misalignment in relation to the physical size of the detector.

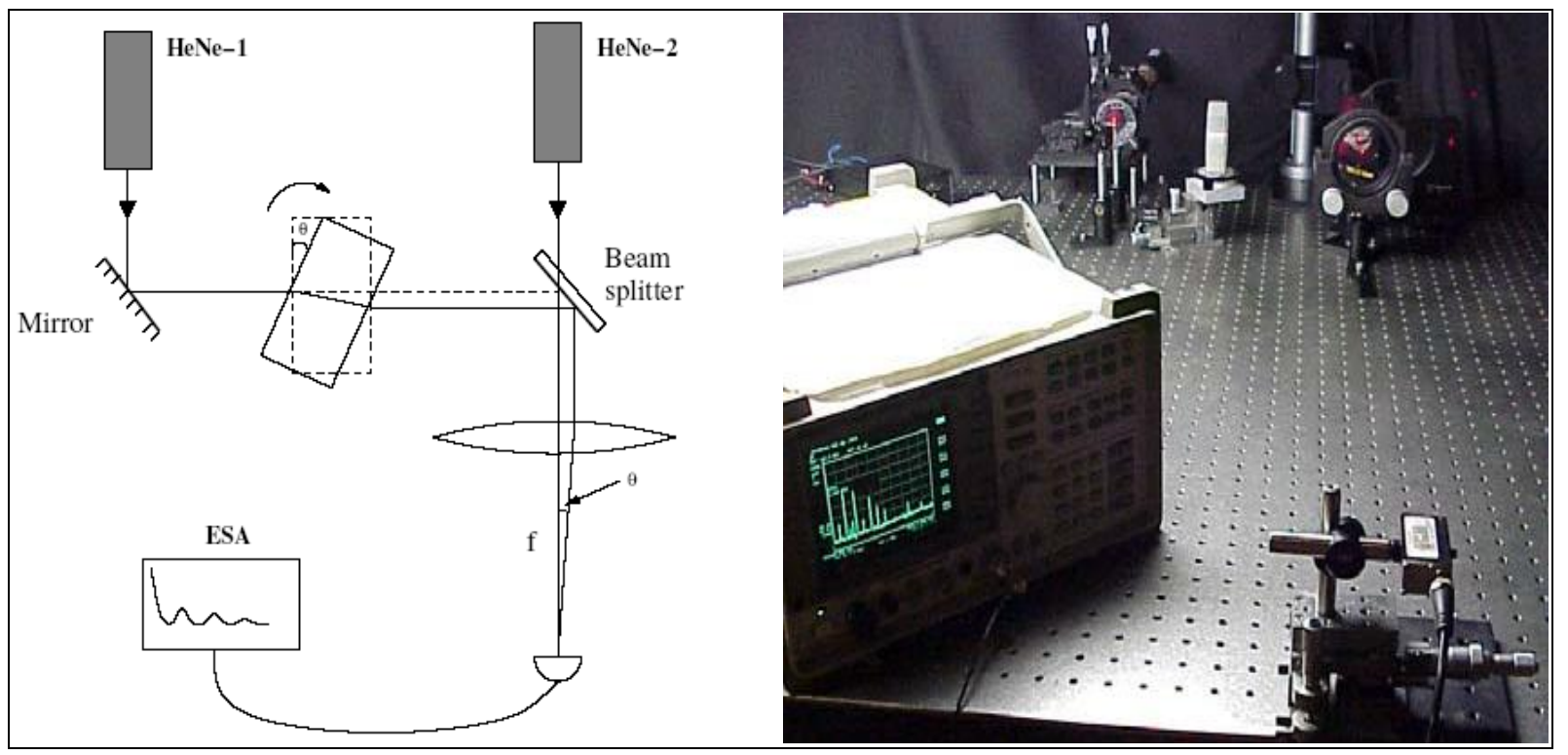

Figure 7. An optical experiment demonstrating angular sensitivity in heterodyne sensing. The electronic spectral analyzer recorded the resultant beams from two He-Ne lasers superposed on a detector. A glass block mounted on a rotating mount introduced the angular misalignment [6].

\section{SUMMARY AND CONCLUSIONS}

In this paper, the misalignment of heterodyne beams as in the case of a coherent lidar is examined in the light of NIW property. The NIW property states that in the linear domain, all waves pass through each other unperturbed. The superposition effects due to light beams become manifest through the response characteristics of the detecting dipoles. Finite amount of beam non-collinearity in the design of coherent lidar can be tolerated at the cost of some signal strength. Similarly, Finite amount of phase-front mismatch can also be tolerated at the cost of signal strength since it is 
equivalent to beam non-collinearity. Spectrally "incoherent" source can also be detected and analyzed by heterodyne method by judicious choice of a tunable LO and band limited detector and/or electronics. Allowed quantum properties and restrictions of the detecting dipole molecules (single or collective) should be explored more deeply to understand the optical superposition phenomenon.

\section{REFERENCES}

[1] Chandra Roychoudhari, "Principle of non-interaction of Waves," Journal of Nanophotonics, Vol. 4, 043512 (2 July 2010).

[2] T. Fujii and T. Fukuchi, (ed.), Laser Remote Sensing, Taylor and Francis, New York, 2005.

[3] C. Roychoudhuri, Phys. Essays 19 (3), September 2006; "Locality of superposition principle is dictated by detection processes".

[4] Michael Ambroselli and Chandrasekhar Roychoudhuri "Visualizing superposition process and appreciating the principle of non-interaction of waves", Proc. SPIE 8121, The Nature of Light: What are Photons? IV, 81211F (September 28, 2011); doi:10.1117/12.893565.

[5] C. Roychoudhuri, Causal Physics: Photon Model from Non-Interaction of Waves, CRC, Oct.2013.

[6] C. Roychoudhuri and N. Prasad, "A deeper look at the fundamentals of heterodyne detection requirements"; Proc. 14th Coherent Laser Radar Conference, Snowmass, Colorado; July 8 -13, 2007. 